\title{
The Electromagnetic Counterpart of the Binary Neutron Star Merger LIGO/Virgo GW170817. II. UV, Optical, and Near-infrared Light Curves and Comparison to Kilonova Models
}

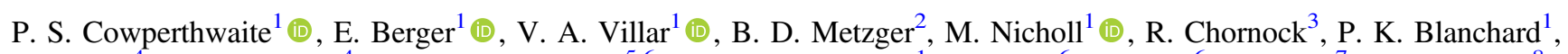

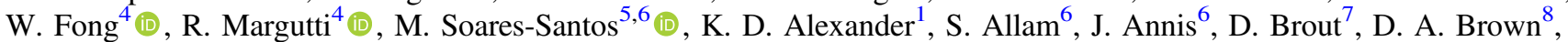
R. E. Butler ${ }^{6,9}$, H.-Y. Chen ${ }^{10}$, H. T. Diehl ${ }^{6}$, Z. Doctor ${ }^{11}$, M. R. Drout ${ }^{12,76}$, T. Eftekhari ${ }^{1}$, B. Farr ${ }^{11,13,14}$, D. A. Finley ${ }^{6}$, R. J. Foley ${ }^{15}$, J. A. Frieman ${ }^{6,13}$, C. L. Fryer ${ }^{16}$, J. García-Bellido ${ }^{17}$, M. S. S. Gill ${ }^{18}$, J. Guillochon ${ }^{1}$, K. Herner ${ }^{6}$, D. E. Holz ${ }^{13,19}$, D. Kasen ${ }^{20,21}$, R. Kessler ${ }^{10,13}$, J. Marriner ${ }^{6}$, T. Matheson ${ }^{22}$, E. H. Neilsen, Jr. ${ }^{6}$, E. Quataert ${ }^{23}$, A. Palmese ${ }^{24}$, A. Rest ${ }^{25,26}$, M. Sako ${ }^{7}$, D. M. Scolnic ${ }^{13}$, N. Smith ${ }^{27}$, D. L. Tucker ${ }^{6}$, P. K. G. Williams ${ }^{1}$, E. Balbinot ${ }^{28}$, J. L. Carlin ${ }^{29}$, E. R. Cook ${ }^{30}$, F. Durret ${ }^{31}$, T. S. Li ${ }^{6}$, P. A. A. Lopes $^{32}$, A. C. C. Lourenço ${ }^{32}$, J. L. Marshall ${ }^{30}$, G. E. Medina ${ }^{33}$, J. Muir ${ }^{34}$, R. R. Muñoz ${ }^{33}$, M. Sauseda ${ }^{30}$, D. J. Schlegel ${ }^{35}$, L. F. Secco ${ }^{7}$, A. K. Vivas ${ }^{36}$, W. Wester ${ }^{6}$, A. Zenteno ${ }^{36}$, Y. Zhang ${ }^{6}$, T. M. C. Abbott ${ }^{36}$, M. Banerji ${ }^{37,38}$, K. Bechtol ${ }^{29}$, A. Benoit-Lévy ${ }^{24,39,40}$, E. Bertin ${ }^{39,40}$, E. Buckley-Geer ${ }^{6}$, D. L. Burke ${ }^{18,41}$, D. Capozzi ${ }^{42}$, A. Carnero Rosell ${ }^{43,44}$, M. Carrasco Kind ${ }^{45,46}$, F. J. Castander ${ }^{47}$, M. Crocce ${ }^{47}$, C. E. Cunha ${ }^{18}$, C. B. D'Andrea ${ }^{7}$, L. N. da Costa ${ }^{43,44}$, C. Davis ${ }^{18}$, D. L. DePoy ${ }^{30}$, S. Desai ${ }^{48}$, J. P. Dietrich ${ }^{49,50}$, A. Drlica-Wagner ${ }^{6}$, T. F. Eifler ${ }^{51,52}$, A. E. Evrard ${ }^{53,54}$, E. Fernandez ${ }^{55}$, B. Flaugher ${ }^{6}$, P. Fosalba ${ }^{47}$, E. Gaztanaga ${ }^{47}$, D. W. Gerdes ${ }^{53,54}$, T. Giannantonio ${ }^{37,38,56}$, D. A. Goldstein ${ }^{57,58}$, D. Gruen ${ }^{18,41}$, R. A. Gruendl ${ }^{45,46}$,

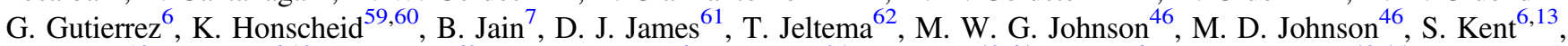

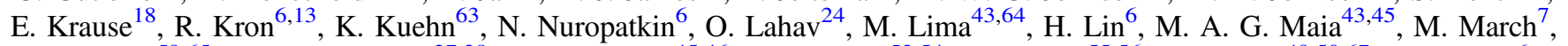
P. Martini ${ }^{59,65}$, R. G. McMahon ${ }^{37,38}$, F. Menanteau ${ }^{45,46}$, C. J. Miller ${ }^{53,54}$, R. Miquel ${ }^{55,56}$, J. J. Mohr ${ }^{49,50,67}$, E. Neilsen ${ }^{6}$,

R. C. $\mathrm{Nichol}^{42}$, R. L. C. Ogando ${ }^{43,44}$, A. A. Plazas ${ }^{52}$, N. Roe ${ }^{58}$, A. K. Romer ${ }^{68}$, A. Roodman ${ }^{18,41}$, E. S. Rykoff ${ }^{18,41}$, E. Sanchez ${ }^{69}$, V. Scarpine ${ }^{6}$, R. Schindler ${ }^{41}$, M. Schubnell ${ }^{54}$, I. Sevilla-Noarbe ${ }^{69}$, M. Smith ${ }^{70}$, R. C. Smith ${ }^{36}$, F. Sobreira ${ }^{43,71}$, E. Suchyta ${ }^{72}$, M. E. C. Swanson ${ }^{46}$, G. Tarle ${ }^{54}$, D. Thomas ${ }^{42}$, R. C. Thomas ${ }^{58}$, M. A. Troxel $^{59,60}$, V. Vikram ${ }^{73}$, A. R. Walker ${ }^{36}$, R. H. Wechsler ${ }^{18,41,74}$, J. Weller ${ }^{49,56,67}$, B. Yanny ${ }^{6}$, and J. Zuntz ${ }^{75}$

\footnotetext{
${ }^{1}$ Harvard-Smithsonian Center for Astrophysics, 60 Garden Street, Cambridge, Massachusetts 02138, USA

${ }^{2}$ Department of Physics and Columbia Astrophysics Laboratory, Columbia University, New York, NY 10027, USA ${ }^{3}$ Astrophysical Institute, Department of Physics and Astronomy, 251B Clippinger Lab, Ohio University, Athens, OH 45701, USA

${ }^{4}$ CIERA and Department of Physics and Astronomy, Northwestern University, Evanston, IL 60208, USA ${ }^{5}$ Department of Physics, Brandeis University, Waltham, MA 02454, USA

${ }^{6}$ Fermi National Accelerator Laboratory, P.O. Box 500, Batavia, IL 60510, USA

${ }^{7}$ Department of Physics and Astronomy, University of Pennsylvania, Philadelphia, PA 19104, USA

${ }^{8}$ Department of Physics, Syracuse University, Syracuse, NY 13224, USA

${ }^{9}$ Department of Astronomy, Indiana University, 727 E. Third Street, Bloomington, IN 47405, USA

${ }^{10}$ Department of Astronomy and Astrophysics, University of Chicago, Chicago, IL 60637, USA

${ }^{11}$ Kavli Institute for Cosmological Physics, University of Chicago, Chicago, IL 60637, USA

${ }^{12}$ The Observatories of the Carnegie Institution for Science, 813 Santa Barbara St., Pasadena, CA 91101, USA

${ }_{14}^{13}$ Department of Physics, University of Oregon, Eugene, OR 97403, USA

${ }^{14}$ Enrico Fermi Institute, University of Chicago, Chicago, IL 60637, USA

${ }^{15}$ Department of Astronomy and Astrophysics, University of California, Santa Cruz, CA 95064, USA

${ }^{16}$ Center for Theoretical Astrophysics, Los Alamos National Laboratory, Los Alamos, NM 87544, USA

${ }^{17}$ Instituto de Física Teórica CSIC/UAM, Universidad Autónoma de Madrid, Cantoblando E-28049 Madrid, Spain

${ }^{18}$ Kavli Institute for Particle Astrophysics \& Cosmology, P.O. Box 2450, Stanford University, Stanford, CA 94305, USA

${ }^{19}$ Enrico Fermi Institute, Department of Physics, Department of Astronomy and Astrophysics, 5640 South Ellis Ave, Chicago, IL 60637, USA

${ }^{20}$ Departments of Physics and Astronomy, and Theoretical Astrophysics Center, University of California, Berkeley, CA 94720-7300, USA

${ }^{21}$ Nuclear Science Division, Lawrence Berkeley National Laboratory, Berkeley, CA 94720-8169, USA

${ }^{22}$ National Optical Astronomy Observatory, 950 North Cherry Avenue, Tucson, AZ 85719, USA

${ }^{23}$ Department of Astronomy \& Theoretical Astrophysics Center, University of California, Berkeley, CA 94720-3411, USA

${ }^{24}$ Department of Physics \& Astronomy, University College London, Gower Street, London WC1E 6BT, UK

${ }^{25}$ Space Telescope Science Institute, 3700 San Martin Drive, Baltimore, MD 21218, USA

${ }^{26}$ Department of Physics and Astronomy, The Johns Hopkins University, 3400 North Charles Street, Baltimore, MD 21218, USA

${ }^{27}$ Steward Observatory, University of Arizona, 933 N. Cherry Avenue, Tucson, AZ 85721, USA

${ }^{28}$ Department of Physics, University of Surrey, Guildford GU2 7XH, UK

${ }^{29}$ LSST, 933 North Cherry Avenue, Tucson, AZ 85721, USA

${ }^{30}$ George P. and Cynthia Woods Mitchell Institute for Fundamental Physics and Astronomy, and Department of Physics and Astronomy, Texas A\&M University, College Station, TX 77843, USA

${ }^{31}$ Institut d'Astrophysique de Paris (UMR7095: CNRS \& UPMC), 98 bis Bd Arago, F-75014, Paris, France

32 Observatòrio do Valongo, Universidade Federal do Rio de Janeiro, Ladeira do Pedro Antônio 43, Rio de Janeiro, RJ, 20080-090, Brazil

${ }^{33}$ Departamento de Astronomòa, Universidad de Chile, Camino del Observatorio 1515, Las Condes, Santiago, Chile

${ }^{34}$ Department of Physics, University of Michigan, 450 Church St, Ann Arbor, MI 48109-1040, USA

${ }^{35}$ Physics Division, Lawrence Berkeley National Laboratory, Berkeley, CA 94720-8160, USA
${ }^{36}$ Cerro Tololo Inter-American Observatory, National Optical Astronomy Observatory, Casilla 603, La Serena, Chile

${ }^{37}$ Institute of Astronomy, University of Cambridge, Madingley Road, Cambridge CB3 OHA, UK

${ }^{38}$ Kavli Institute for Cosmology, University of Cambridge, Madingley Road, Cambridge CB3 OHA, UK

${ }^{39}$ CNRS, UMR 7095, Institut d'Astrophysique de Paris, F-75014, Paris, France

${ }^{40}$ Sorbonne Universités, UPMC Univ Paris 06, UMR 7095, Institut d'Astrophysique de Paris, F-75014, Paris, France ${ }^{41}$ SLAC National Accelerator Laboratory, Menlo Park, CA 94025, USA

${ }^{42}$ Institute of Cosmology \& Gravitation, University of Portsmouth, Portsmouth PO1 3FX, UK
} 


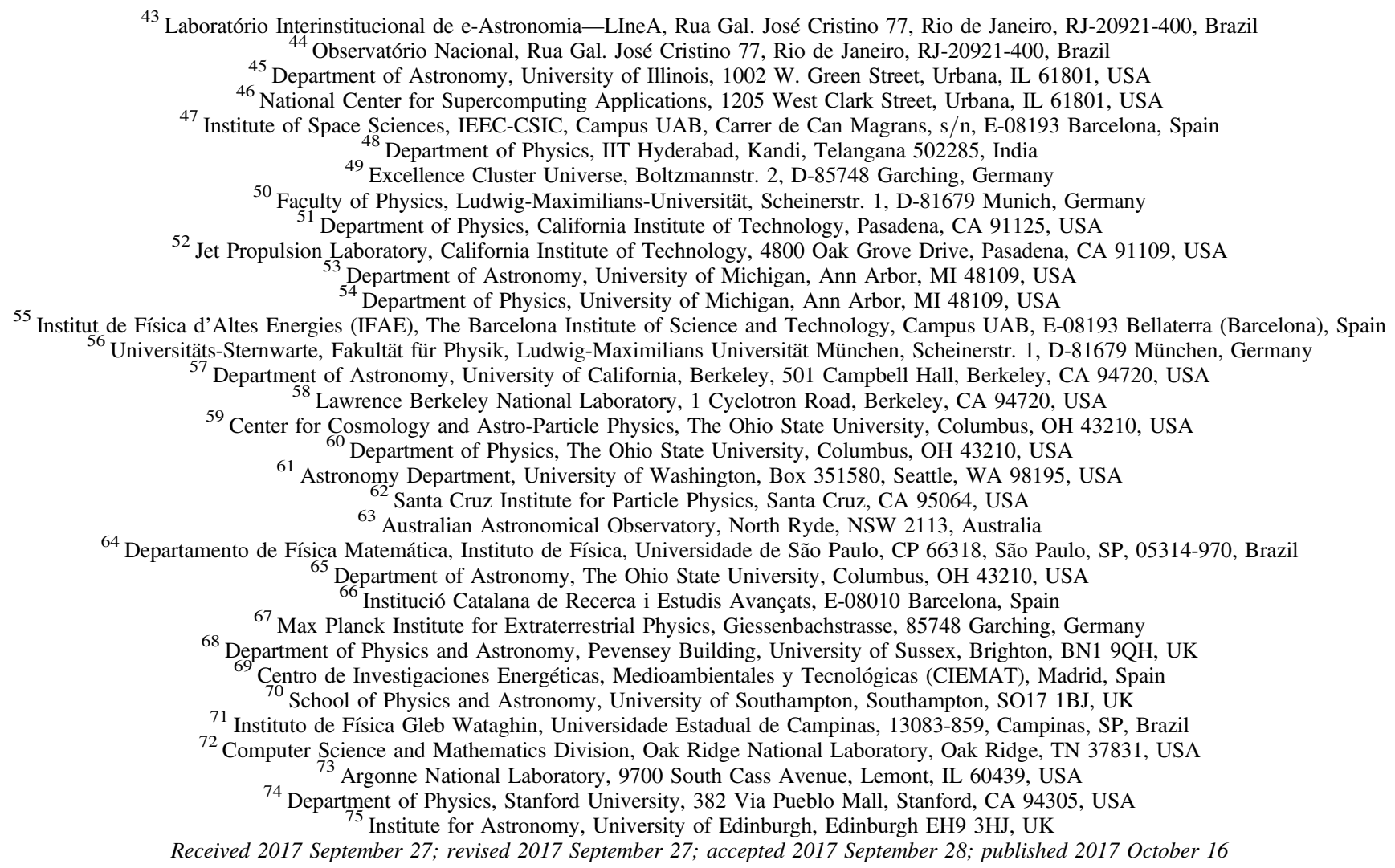

\begin{abstract}
We present UV, optical, and near-infrared (NIR) photometry of the first electromagnetic counterpart to a gravitational wave source from Advanced Laser Interferometer Gravitational-wave Observatory (LIGO)/Virgo, the binary neutron star merger GW170817. Our data set extends from the discovery of the optical counterpart at 0.47-18.5 days post-merger, and includes observations with the Dark Energy Camera (DECam), Gemini-South/ FLAMINGOS-2 (GS/F2), and the Hubble Space Telescope (HST). The spectral energy distribution (SED) inferred from this photometry at 0.6 days is well described by a blackbody model with $T \approx 8300 \mathrm{~K}$, a radius of $R \approx 4.5 \times 10^{14} \mathrm{~cm}$ (corresponding to an expansion velocity of $v \approx 0.3 c$ ), and a bolometric luminosity of $L_{\text {bol }} \approx 5 \times 10^{41} \mathrm{erg} \mathrm{s}^{-1}$. At 1.5 days we find a multi-component SED across the optical and NIR, and subsequently we observe rapid fading in the UV and blue optical bands and significant reddening of the optical/ NIR colors. Modeling the entire data set, we find that models with heating from radioactive decay of ${ }^{56} \mathrm{Ni}$, or those with only a single component of opacity from $r$-process elements, fail to capture the rapid optical decline and red optical/NIR colors. Instead, models with two components consistent with lanthanide-poor and lanthanide-rich ejecta provide a good fit to the data; the resulting "blue" component has $M_{\mathrm{ej}}^{\text {blue }} \approx 0.01 M_{\odot}$ and $v_{\mathrm{ej}}^{\text {blue }} \approx 0.3 \mathrm{c}$, and the "red" component has $M_{\mathrm{ej}}^{\mathrm{red}} \approx 0.04 M_{\odot}$ and $\nu_{\mathrm{ej}}^{\mathrm{red}} \approx 0.1 \mathrm{c}$. These ejecta masses are broadly consistent with the estimated $r$-process production rate required to explain the Milky Way $r$-process abundances, providing the first evidence that binary neutron star (BNS) mergers can be a dominant site of $r$-process enrichment.
\end{abstract}

Key words: binaries: close - catalogs - gravitational waves - stars: neutron - surveys

\section{Introduction}

The era of gravitational wave (GW) astronomy began on 2015 September 14 when the Advanced Laser Interferometer Gravitational-wave Observatory (LIGO) made the first direct detection of gravitational waves, resulting from the merger of a stellar mass binary black hole (BBH; GW150914; Abbott et al. 2016a). LIGO has since announced the detection of three additional BBH events (Abbott et al. 2016b, 2017a; LIGO Scientific Collaboration et al. 2017). There are currently no robust theoretical predictions for electromagnetic (EM) emission associated with such mergers.

\footnotetext{
${ }^{76}$ Hubble, Carnegie-Dunlap Fellow.
}

By contrast, mergers involving at least one neutron star can produce a wide range of EM signals, spanning from gamma-rays to radio (e.g., Metzger \& Berger 2012). In the optical/nearinfrared (NIR) bands, the most promising counterpart is the kilonova $(\mathrm{KN})$, a roughly isotropic thermal transient powered by the radioactive decay of rapid neutron capture ( $r$-process) elements synthesized in the merger ejecta (Li \& Paczyński 1998; Metzger et al. 2010; Roberts et al. 2011; Metzger \& Berger 2012; Barnes \& Kasen 2013; Tanaka \& Hotokezaka 2013). The properties of the KN emission (luminosity, timescale, spectral peak) depend sensitively on the ejecta composition. For ejecta containing Fe-group or light $r$-process nuclei with atomic 
mass number $A \lesssim 140$, the $\mathrm{KN}$ emission is expected to peak at optical wavelengths at a luminosity $L_{\mathrm{p}} \sim 10^{41}-10^{42} \mathrm{erg} \mathrm{s}^{-1}$ on a short timescale of $t_{\mathrm{p}} \sim 1$ day (a so-called "blue" KN; Metzger et al. 2010; Roberts et al. 2011; Metzger \& Fernández 2014). By contrast, for ejecta containing heavier lanthanide elements $(A \gtrsim 140)$ the emission is predicted to peak at NIR wavelengths with $L_{\mathrm{p}} \sim 10^{40}-10^{41} \mathrm{erg} \mathrm{s}^{-1}$ over a longer timescale of $t_{\mathrm{p}} \sim 1$ week (a so-called "red" KN; Barnes \& Kasen 2013; Kasen et al. 2013; Tanaka \& Hotokezaka 2013).

The first direct detection of gravitational waves from the inspiral and merger of a binary neutron star (BNS) was made on 2017 August 17 (GW170817; LIGO Scientific Collaboration \& Virgo Collaboration 2017a, 2017b, 2017c). This source was coincident with a short burst of gamma-rays detected by both Fermi/Gamma-ray Burst Monitor (GBM) and INTEGRAL (GRB 170817A; Blackburn et al. 2017; Goldstein et al. 2017a, 2017b; Savchenko et al. 2017a, 2017b; von Kienlin et al. 2017). Rapid optical follow-up by our Dark Energy Camera (DECam) program (Flaugher et al. 2015), starting just $11.4 \mathrm{hr}$ after the GW trigger, led to the discovery of an associated optical counterpart in the nearby $(d \approx 39.5 \mathrm{Mpc}$; Freedman et al. 2001) galaxy NGC 4993 (Allam et al. 2017; Soares-Santos et al. 2017). This optical source was independently discovered by several groups (Abbott et al. 2017b), and first announced as SSS17a by Coulter et al. (2017a, 2017b). The source has also been independently named DLT17ck (Valenti et al. 2017; Yang et al. 2017) and AT2017gfo.

Here we present rapid-cadence UV, optical, and NIR observations spanning from the time of discovery to 18.5 days post-merger. We construct well-sampled light curves and spectral energy distributions (SEDs) using data from DECam, along with Gemini-South/FLAMINGOS-2 (GS/F2) and Hubble Space Telescope (HST). We show that the data cannot be fit by a model with heating from ${ }^{56} \mathrm{Ni}$ radioactive decay and Fe-peak opacities (as in normal supernovae), but instead requires heating from $r$-process nuclei and at least two components consistent with lanthanide-poor and lanthaniderich opacities. We further use the data to determine the ejecta masses and velocities for each component.

All magnitudes presented in this work are given in the $A B$ system and corrected for Galactic reddening ${ }^{77}$ with $E(B-V)=0.105$, applying the calibration of Schlafly \& Finkbeiner (2011). We assume a negligible reddening contribution from the host (Blanchard et al. 2017).

\section{Observations and Data Analysis}

A summary of the observations and photometry described in this section is available in the Appendix.

\subsection{DECam}

We processed all of the DECam images using the photpipe pipeline (e.g., Rest et al. 2005, 2014) in order to perform single-epoch image processing and image subtraction using the hotpants software package (Becker 2015). Pointspread function (PSF) photometry was performed on the subtracted images using an implementation of DoPhot optimized for difference images (Schechter et al. 1993). We performed astrometric and photometric calibration relative to

\footnotetext{
77 This is computed from http://irsa.ipac.caltech.edu/applications/DUST/ using the coordinate transients in Soares-Santos et al. (2017).
}

the Pan-STARRS $1 / 3 \pi$ catalog (PS $1 / 3 \pi$; Chambers et al. 2016), with appropriate corrections between magnitude systems (Scolnic et al. 2015). The typical calibration error is on the order of $\approx 3 \%$. Image subtraction was performed using stacked images from the PS $1 / 3 \pi$ survey as reference images for $g r$-band. DECam images from 2017 August 25 and 2017 August 31 were used as reference images for $u$-band and $i z Y$ band, respectively, after the transient had faded away.

\section{2. $H S T$}

We obtained HST Target-of-Opportunity observations of GW170817 on 2017 August 27.28 (9.8 days post-trigger) UT using ACS/WFC with the F475W, F625W, F775W, and F850LP filters, WFC3/UVIS with the F336W filter, and WFC3/IR with the F160W and F110W filters (PID: 15329; PI: Berger). We retrieved the calibrated data from the Mikulski Archive for Space Telescopes and used the DrizzlePac ${ }^{78}$ software package to create final drizzled images from the individual dithered observations in each filter. We used the astrodrizzle task to correct for optical distortion and improve the resolution from that sampled by the instrumental PSF. We measure the flux of the optical counterpart by fitting a model PSF, constructed from multiple stars in each image, using a custom Python wrapper for DAOPhot (Stetson 1987). We removed contaminating flux from the host galaxy at the transient location using local background subtraction. After subtraction, the typical contribution from the host flux is $\lesssim 5 \%$. We calibrated the photometry for each image using the zeropoints provided by the HST analysis team. ${ }^{79}$

\section{3. $G S / F 2$}

We obtained several epochs of $H K_{s}$ band photometry using FLAMINGOS- 2 on the Gemini-South $8 \mathrm{~m}$ telescope (Eikenberry et al. 2012) starting on 2017 August 19.00 (1.47 days postmerger). We processed the images using standard procedures in the gemini IRAF $^{80}$ package. We created an average sky exposure from the individual dithered frames and then scaled and subtracted from each science image prior to the registration and combination of the images. We performed PSF photometry using field stars and host galaxy subtraction as described in Section 2.2, and calibrated the photometry relative to the 2MASS point source catalog. ${ }^{81}$

\subsection{Swift/UVOT}

The UVOT instrument on board Swift (Gehrels et al. 2004; Roming et al. 2005) began observing the field of the optical counterpart on 2017 August 18.167 UT with the $U, W 1, W 2$, and M2 filters (Cenko et al. 2017; Evans et al. 2017a, 2017b). We used the latest HEAsoft release (v6.22) with the corresponding calibration files and updated zeropoints in order to independently analyze the data. We performed photometry in a $3^{\prime \prime}$ photometric aperture to in order minimize the contamination from host galaxy light, following the prescriptions by Brown et al. (2009). We estimated and subtracted the contribution from host galaxy light using deep UVOT

\footnotetext{
${ }^{78}$ http://drizzlepac.stsci.edu/

79 http://www.stsci.edu/hst/acs/analysis/zeropoints

${ }^{80}$ IRAF is distributed by the National Optical Astronomy Observatory, which is operated by the Association of Universities for Research in Astronomy (AURA) under a cooperative agreement with the National Science Foundation. 81 https://www.ipac.caltech.edu/2mass/
} 

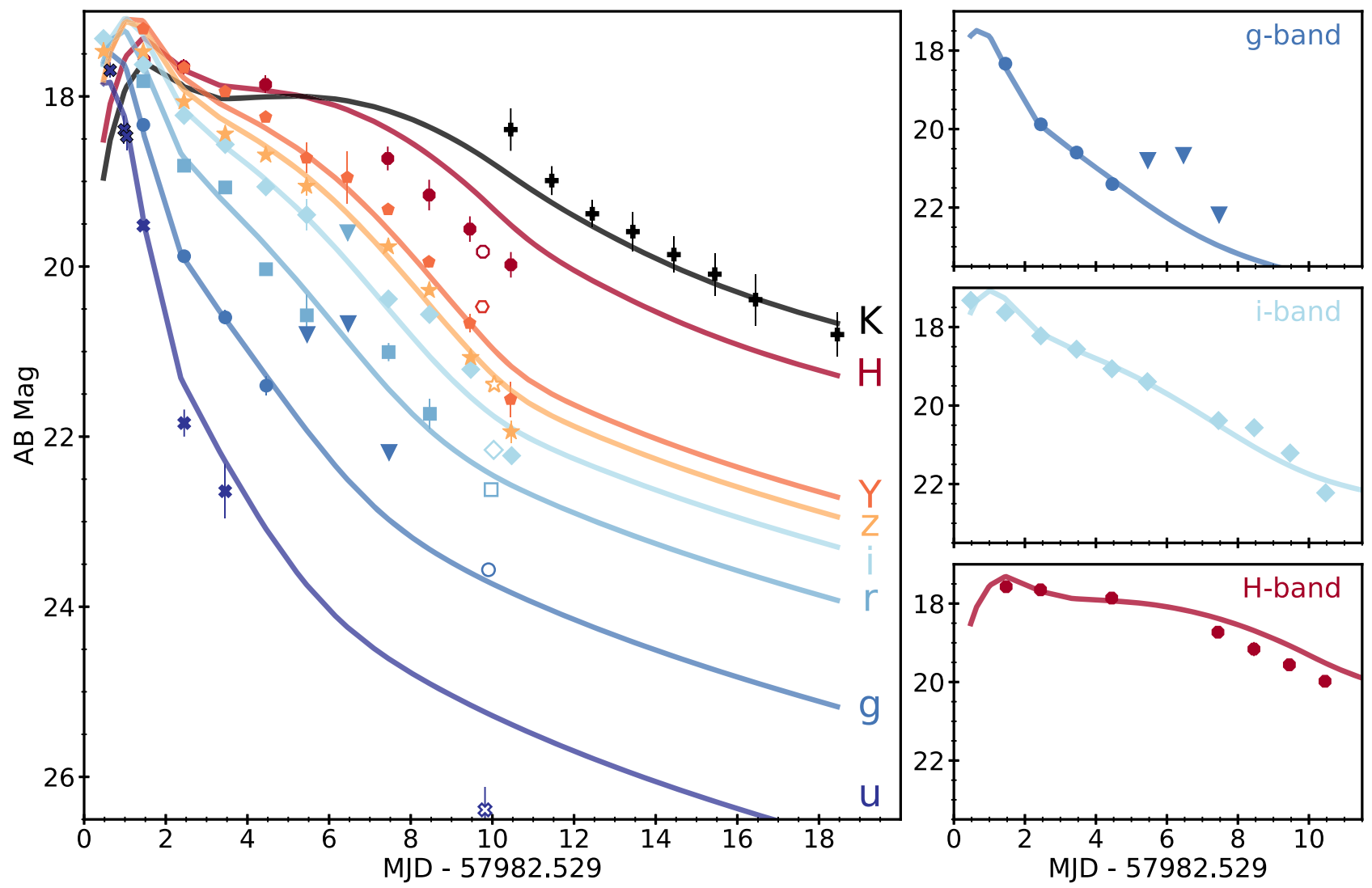

Figure 1. UV, optical, and NIR light curves of the counterpart of GW170817. The two-component model for $r$-process heating and opacities (Section 4) is shown as solid lines. The right panels focus on the $g$ (top), $i$ (middle), and $H$-band photometry (bottom) over the first 10 nights. Triangles represent $3 \sigma$ upper limits. Error bars are given at the $1 \sigma$ level in all panels, but may be smaller than the points.

observations acquired at later times, when the UV emission from the transient was no longer present in the images (Swift ID 07012979003). The systematic effect from the host light contamination is $\approx 3 \%$ (see e.g., Brown et al. 2009).

\section{Light Curves and Spectral Energy Distributions}

\subsection{Light Curves}

Our UV/optical/NIR light curves are shown in Figure 1. The data span from 0.47 to 18.5 days post-merger, with bluer bands fading below the detection limits at earlier times. The light curve coverage was truncated by the proximity of the source to the Sun. We first note that the light curves are not well described by a power law, indicating minimal contribution from a GRB optical afterglow over the timescale of our observations. This is consistent with modeling of the afterglow based on X-ray and radio observations (Margutti et al. 2017; Alexander et al. 2017).

The light curves exhibit a rapid decline in the bluest bands $(u g)$, an intermediate decline rate in the red optical bands $(r i z Y)$, and a shallow decline in the NIR $\left(H K_{s}\right)$. However, while the $u$ - and $g$-band light curves decline by $\approx 2$ mag day $^{-1}$ starting with the earliest observations, the redder optical bands exhibit a more complex behavior: they exhibit a comparatively slow decline $\left(\approx 0.3 \mathrm{mag} \mathrm{day}^{-1}\right)$ over the first 1.5 days, develop a shoulder at about 4 days, and subsequently begin to decline at about 8 days.
We find a similar rapid evolution in the colors of the transient (Figure 2). In particular, the $u-g$ and $g-r$ colors become redder by about 1 mag between about 1.5 and 3.5 days. The colors in the redder optical bands exhibit slower evolution, with $r-i \approx 0.5-1 \mathrm{mag}, i-z \approx 0-0.5 \mathrm{mag}$, and $z-Y \approx 0.3 \mathrm{mag}$. These colors are significantly redder than those of known supernovae near explosion (e.g., Folatelli et al. 2010; Bianco et al. 2014; Galbany et al. 2016).

\subsection{Spectral Energy Distribution}

We construct SEDs from photometry at several epochs from about 0.6 to 10 days post-merger (Figure 2). The SEDs exhibit rapid evolution from an initial peak at $\sim 3500 \AA$ to a final peak at $\gtrsim 15000 \AA$ by 10 days. Moreover, the SED at 1.5 days appears to consist of two components, as indicated by the changing slope in the NIR emission. The same rapid evolution and structure are apparent in the optical and NIR spectra at comparable epochs (Chornock et al. 2017; Nicholl et al. 2017).

The SED at 0.6 days is well described by a blackbody with $T \sim 8300 \mathrm{~K}$ and $R \sim 4.5 \times 10^{14} \mathrm{~cm}$, corresponding to an expansion velocity of $v \sim 0.3 c$. This is somewhat larger than the velocities observed in broad-lined $\mathrm{SNe}$ Ic (for which $v \approx 0.1 c$; Modjaz et al. 2016), but is consistent with expectations for ejecta resulting from a BNS merger (Metzger 2017). The SEDs at later times are not well described by a blackbody curve, instead exhibiting strong flux suppression at 

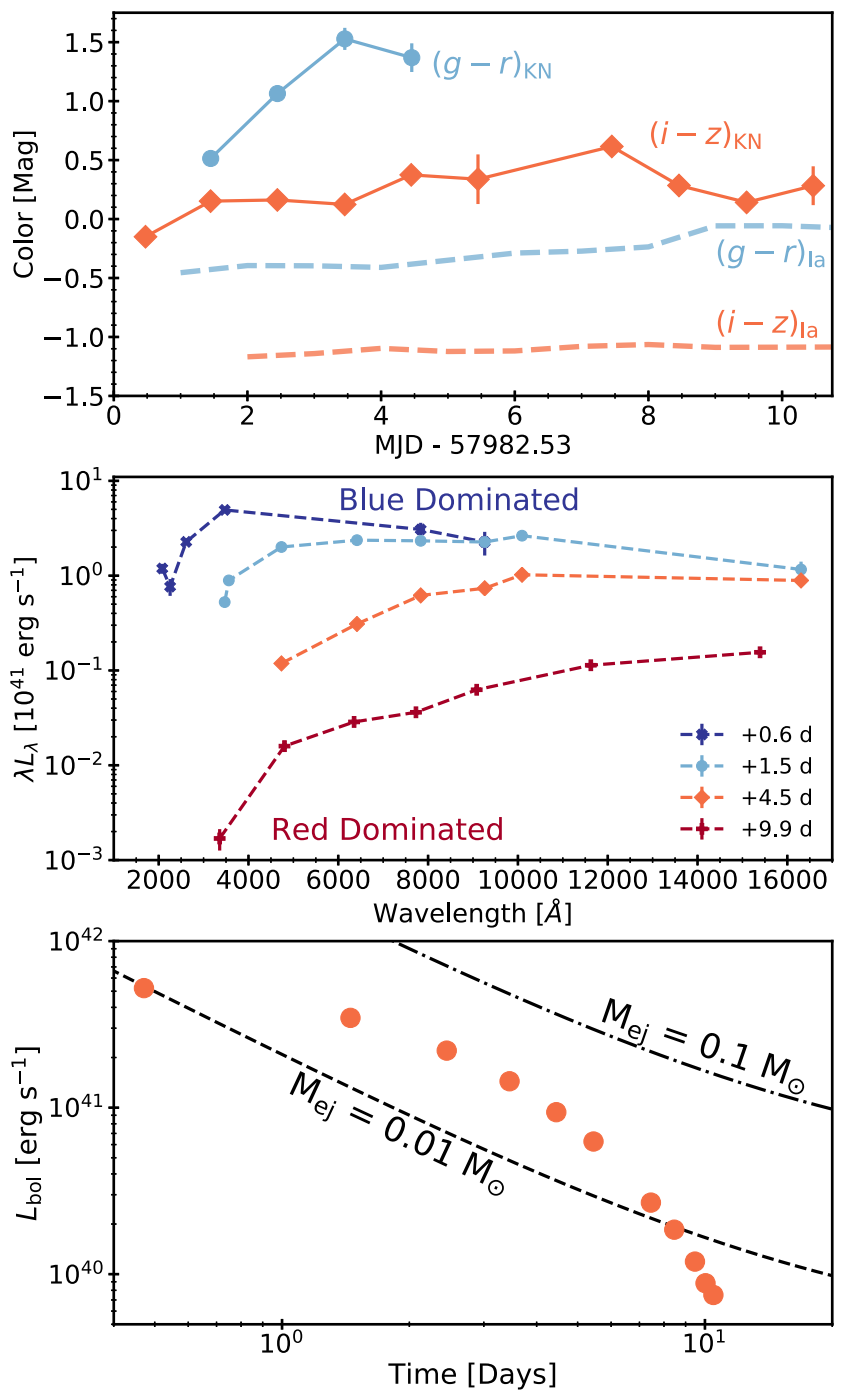

Figure 2. Top: optical colors from DECam observations as a function of time. We observed rapid and early reddening in $g-r$ compared to the relatively flat but red $i-z$ colors. Also shown are template Ia SN colors relative to the explosion for comparison (Nugent et al. 2002). Middle: SEDs at four representative epochs (assuming isotropic emission). The transition from a blue dominated spectrum at early times to a spectrum dominated by a red component at late times is clearly visible. Bottom: Bolometric light curve spanning ugrizYH. Expected values for $r$-process heating from Metzger et al. (2010) are shown for comparison, indicating that the observed emission requires few $\times 10^{-2} M_{\odot}$ of $r$-process ejecta. Error bars are given at the $1 \sigma$ level in all panels, but may be smaller than the points.

blue wavelengths that leads to a spectrum with a sharper peak than a blackbody. This behavior is also present in our optical spectra (Nicholl et al. 2017).

\subsection{Bolometric Light Curve}

We construct a bolometric light curve from the ugriz $Y H$ data spanning 11 days. We fit the time evolution in each band independently with a linear model and interpolate the magnitudes to a common grid of times. The bolometric luminosity is determined using the integrated total flux at each time step; see Figure 2. The peak bolometric luminosity of $\sim 5 \times 10^{41} \mathrm{erg} \mathrm{s}^{-1}$ at 0.6 days is broadly consistent with the luminosity predicted for $r$-process heating by a few $\times 10^{-2} M_{\odot}$ ejecta, similar to the original predictions of Metzger et al. (2010) for blue KN emission from Fe-opacity ejecta. The total radiated energy during the first $\sim 10$ days is $\approx 10^{47} \mathrm{erg}$.

\subsection{Qualitative Comparisons to Kilonova Emission}

Before exploring detailed models, there are several lines of preliminary evidence that suggest the optical counterpart is an $r$-process-powered KN. The presence of an initially blue SED, which then transitions to a multi-component SED and finally to a red SED, is strongly suggestive of both blue and red KN emission; this is consistent with lanthanide-poor and rich ejecta components, respectively (Metzger et al. 2010; Barnes \& Kasen 2013; Tanaka \& Hotokezaka 2013; Metzger \& Fernández 2014; Kasen et al. 2015; Wollaeger et al. 2017). Furthermore, the deviations from a pure blackbody spectrum at late times are indicative of the strong UV line blanketing expected for lanthanide-rich material, lending further evidence to the existence of a red $\mathrm{KN}$ component. This behavior is also seen in optical/NIR spectra of the transient (Chornock et al. 2017; Nicholl et al. 2017).

The fact that this red component does not initially obscure the emission from a blue component suggests that we require two separate emitting regions with distinct sources of ejecta. If the KN outflow is quasi-spherical, then the blue component must reside outside of the material with red emission. Alternatively, if the outflow is not spherically symmetric, the blue and red ejecta should occupy distinct portions of the outflowing solid angle. This feature is suggested in several models that consider lanthanide-rich material ejected in the equatorial plane while the lanthanide-poor material is ejected from the polar regions (Kasen et al. 2015; Metzger 2017).

\section{Kilonova Modeling}

We test the conjecture that the UV/optical/NIR transient is an $r$-process $\mathrm{KN}$ by fitting several isotropic, one-zone, gray opacity models to the light curves. For each model, we assume a blackbody SED which evolves assuming a constant ejecta velocity until it has reached a minimum temperature, at which point the photosphere has receded into the ejecta and the temperature no longer evolves. A similar temperature "floor" is predicted in Barnes \& Kasen (2013), and we include this minimum temperature as a fitted parameter. We additionally fit for a "scatter" term, added in quadrature to all photometric errors, which roughly accounts for additional systematic uncertainties that are not included in our model.

We use MOSFiT ${ }^{82}$ (Guillochon et al. 2017; Nicholl et al. 2017), an open-source light curve fitting tool that utilizes a Markov Chain Monte Carlo (MCMC) to sample the model posterior. For each model, we ensure convergence by enforcing a Gelman-Rubin statistic $<1.1$ (Gelman \& Rubin 1992). We compare models using the Watanabe-Akaike Information Criteria (WAIC; Watanabe 2010; Gelman et al. 2014), which accounts for both the likelihood score and the number of fitted parameters. The best-fit parameters, uncertainties, and WAIC scores for each model are provided in Table 1.

We first attempt a simple supernova model, namely heating by the radioactive decay of ${ }^{56} \mathrm{Ni}$ and Fe-peak opacity of

\footnotetext{
82 https://github.com/guillochon/MOSFiT
} 


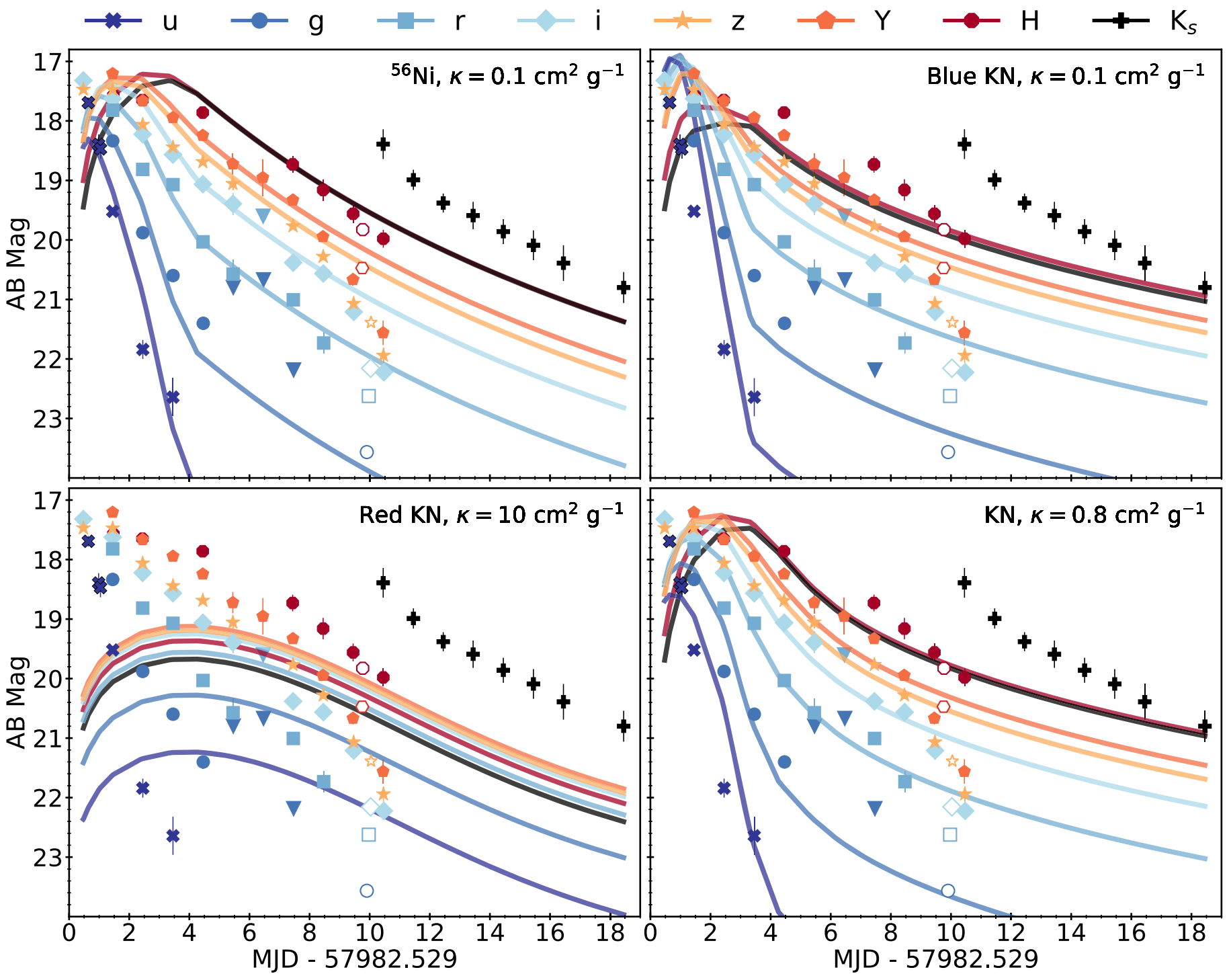

Figure 3. Top left: fitting the data with a Type I b/c SN model powered by the radioactive decay of ${ }^{56} \mathrm{Ni}$. This model clearly fails to capture the late-time NIR behavior and requires an unphysically large fraction of the ejecta to be synthesized into nickel ( $\sim 75 \%)$. Top right: fitting the data with a single-component "blue" KN model. Like the SN model, this fit is unable to capture the late-time NIR behavior and overall spectra shape. Bottom left: fitting the data with a single-component "red" KN model. This model clearly fails to capture any of the observed behavior. Bottom right: fitting the data with a single-component KN model with the opacity as a free parameter. Again, this model fails to capture the late-time NIR behavior. This is suggestive of the fact that we need to model multiple ejecta components simultaneously. Error bars are given at the $1 \sigma$ level in all panels, but may be smaller than the points.

$\kappa=0.1 \mathrm{~cm}^{2} \mathrm{~g}^{-1}$ (see Villar et al. 2017). The model parameters are the ejecta mass and velocity, and the ${ }^{56} \mathrm{Ni}$ mass fraction in the ejecta (as well as the temperature floor and scatter). The best-fit model has $M_{\mathrm{ej}} \approx 0.01 M_{\odot}, v_{\mathrm{ej}} \approx 0.26 \mathrm{c}$, and $f^{\mathrm{Ni}} \approx 0.75$. The parameters are comparable to those we inferred from blackbody fits to the flux and SEDs in the previous section, but the overall fit is poor. In particular, this model severely underestimates the NIR light curves, while the high ${ }^{56} \mathrm{Ni}$ fraction is inconsistent with the optical spectra (Nicholl et al. 2017). We therefore conclude that the transient is not powered by the radioactive decay of ${ }^{56} \mathrm{Ni}$.

We next turn to $r$-process heating, using the model outlined in Metzger (2017) and implemented in Villar et al. (2017). This model includes the ejecta mass, ejecta velocity, and opacity as fitted parameters (as well as the temperature floor and scatter). Within this context we first assume an Fe-peak opacity of $\kappa=0.1 \mathrm{~cm}^{2} \mathrm{~g}^{-1}$ (our "blue" model; e.g., as assumed historically in Li \& Paczyński 1998) and fit for the ejecta mass and velocity. This model, with $M_{\mathrm{ej}} \approx 0.03 M_{\odot}$ and $v_{\mathrm{ej}} \approx 0.18 \mathrm{c}$, adequately describes the early light curves $(\lesssim 3$ days), but again is a poor fit to the NIR light curves. More recent calculations indicate that lanthanide-rich ejecta are expected to have a much higher opacity of $\kappa=10 \mathrm{~cm}^{2}$ $\mathrm{g}^{-1}$, leading to a "red" KN (e.g., Barnes \& Kasen 2013). However, such a model (our "red" model), with best-fit values $M_{\mathrm{ej}} \approx 0.03 M_{\odot}$ and $v_{\mathrm{ej}} \approx 0.27 \mathrm{c}$, produces a poor fit to the data as well. In particular, the model light curves exhibit an initial rise for $\approx 4$ days, in contrast to the observed rapid decline at early times, especially in the UV and blue optical bands. Finally, we allow the opacity to vary as a free parameter, finding a best-fit value of $\kappa \approx 0.82 \mathrm{~cm}^{2} \mathrm{~g}^{-1}$, and an associated $M_{\mathrm{ej}} \approx 0.04 M_{\odot}$ and $v_{\mathrm{ej}} \approx 0.27 \mathrm{c}$. However, this model again 
Table 1

Kilonova Model Fits

\begin{tabular}{|c|c|c|c|c|c|c|c|c|c|c|c|}
\hline Model & $\begin{array}{c}M_{\mathrm{ej}}^{\text {blue }} \\
\left(M_{\odot}\right)\end{array}$ & $\begin{array}{c}v_{\mathrm{ej}}^{\text {blue }} \\
\text { (c) }\end{array}$ & $\begin{array}{c}\kappa^{\text {blue }} \\
\left(\mathrm{cm}^{2} \mathrm{~g}^{-1}\right)\end{array}$ & $\begin{array}{c}M_{\mathrm{ej}}^{\text {purple }} \\
\left(M_{\odot}\right)\end{array}$ & $\begin{array}{c}v_{\mathrm{ej}}^{\text {purple }} \\
\text { (c) }\end{array}$ & $\begin{array}{c}\kappa^{\text {purple }} \\
\left(\mathrm{cm}^{2} \mathrm{~g}^{-1}\right)\end{array}$ & $\begin{array}{l}M_{\mathrm{ej}}^{\mathrm{red}} \\
\left(M_{\odot}\right)\end{array}$ & $\begin{array}{l}v_{\mathrm{ej}}^{\mathrm{red}} \\
(\mathrm{c})\end{array}$ & $\begin{array}{c}\kappa^{\mathrm{red}} \\
\left(\mathrm{cm}^{2} \mathrm{~g}^{-1}\right)\end{array}$ & $f^{\mathrm{Ni}}$ & $\overline{\text { WAIC }}$ \\
\hline 2-Comp & $0.014_{-0.001}^{+0.002}$ & $0.266_{-0.002}^{+0.007}$ & $(0.5)$ & $\cdots$ & $\cdots$ & $\cdots$ & $0.036_{-0.002}^{+0.001}$ & $0.123_{-0.014}^{+0.012}$ & $3.349_{-0.337}^{+0.364}$ & $\cdots$ & -102 \\
\hline${ }^{56} \mathrm{Ni}$ & $0.008_{-0.001}^{+0.007}$ & $0.260_{-0.031}^{+0.034}$ & (0.1) & $\ldots$ & $\ldots$ & $\ldots$ & $\ldots$ & $\ldots$ & $\ldots$ & $0.749_{-0.203}^{+0.214}$ & 17 \\
\hline Blue & $0.032_{-0.004}^{+0.002}$ & $0.180_{-0.002}^{+0.002}$ & (0.1) & $\ldots$ & $\ldots$ & $\ldots$ & $\ldots$ & $\ldots$ & $\ldots$ & $\ldots$ & 17 \\
\hline Red & $\ldots$ & $\ldots$ & $\cdots$ & $\ldots$ & $\ldots$ & $\ldots$ & $0.026_{-0.008}^{+0.010}$ & $0.271_{-0.002}^{+0.008}$ & (10) & $\ldots$ & 153 \\
\hline
\end{tabular}

Note. Model parameters and WAIC scores. Numbers in parentheses indicate fixed parameters of the model. The errors represent the $1 \sigma$ confidence interval. Both the 2-component ("2-Comp") and 3-component ("3-Comp") models have significantly smaller WAIC scores (indicating better fits) compared to the four singlecomponent models.

fails to reproduce the initial rapid decline in the UV, as well as the NIR light curves. We therefore conclude that $r$-process heating with a single value for the opacity cannot explain the observed light-curve evolution and colors. The final light curves for these models can be seen in Figure 3.

Inspired by the multi-component observed SED (Figure 2) and by the failure of single-component models to capture both the early rapid decline and the late-time red colors, we explore two multi-component models: (i) a two-component "blue" $\left(\kappa=0.5 \mathrm{~cm}^{2} \mathrm{~g}^{-1}\right)$ plus "red" ( $\kappa$ as a free parameter) model; and (ii) a three-component "blue" $\left(\kappa=0.5 \mathrm{~cm}^{2} \mathrm{~g}^{-1}\right)$ plus "purple" $\left(\kappa=3 \mathrm{~cm}^{2} \mathrm{~g}^{-1}\right)$ plus "red" $\left(\kappa=10 \mathrm{~cm}^{2} \mathrm{~g}^{-1}\right)$ model. These values were recently shown by Tanaka et al. (2017) to roughly capture the detailed opacity from radiative transfer simulations. For each component, we leave $M_{\mathrm{ej}}$ and $v_{\mathrm{ej}}$ as free parameters.

First, we explore the two-component model (with eight free parameters); we vary the ejecta masses, ejecta velocities, and temperature floors, the red component opacity, and a single scatter term. We find that the "blue" component has $M_{\mathrm{ej}}^{\text {blue }} \approx 0.01 M_{\odot}$ and $v_{\mathrm{ej}}^{\text {blue }} \approx 0.27 \mathrm{c}$ (with errors of roughly $10 \%$ ), in good agreement with our inference from the SED at early times (Section 3.2). The "red" component has a much larger mass of $M_{\mathrm{ej}}^{\text {red }} \approx 0.04 M_{\odot}$ but a slower velocity of $v_{\mathrm{ej}}^{\text {red }} \approx 0.12 \mathrm{c}$. The best-fit opacity of this component is $\kappa \approx 3.3 \mathrm{~cm}^{2} \mathrm{~g}^{-1}$, lower than expected for lanthanide-rich ejecta. We find that most of the parameters are uncorrelated, with the exception of the red component's opacity and ejecta velocity, which have a Pearson correlation coefficient of $\sim 0.67$. The resulting parameters and uncertainties from the MCMC fitting are summarized in Table 1.

For the three-component model (with 10 free parameters) we find similar values for the "blue" component $\left(M_{\mathrm{ej}}^{\text {blue }} \approx 0.01 M_{\odot}\right.$ and $\left.v_{\mathrm{ej}}^{\text {blue }} \approx 0.27 \mathrm{c}\right)$ and the "purple" component $\left(M_{\mathrm{ej}}^{\text {purple }} \approx\right.$ $0.03 M_{\odot}$ and $v_{\mathrm{ej}}^{\text {purple }} \approx 0.11 \mathrm{c}$ ). The "red" component is subdominant with $M_{\mathrm{ej}}^{\text {red }} \approx 0.01 M_{\odot}$ and $v_{\mathrm{ej}}^{\text {red }} \approx 0.16 \mathrm{c}$ ); see Table 1 . These ejecta parameters are consistent with those determined from independent modeling of the optical and NIR spectra (Chornock et al. 2017; Nicholl et al. 2017).

Both sets of models are shown in Figure 1 and are essentially indistinguishable. Both provide a much better fit to the data than the single-component models described above, capturing both the initial blue colors and rapid decline, as well as the later redder colors and NIR light curves. Their similar WAIC scores suggest that neither model is statistically preferred. The two models differ most drastically at $\lesssim 5$ days in the $K_{s}$-band, where the two-component model is doublepeaked, while the three-component model is single peaked. While neither model fully captures every feature of the light curves, it is remarkable that these simplified semi-analytic models produce such high-quality fits over a wide range of wavelength and time.

\section{Implications}

In the multi-component models, we can interpret each component as arising from distinct physical regions within the merger ejecta. In both models, the high velocity of the blue KN ejecta suggests that it originates from the shock-heated polar region created when the neutron stars collide (e.g., Oechslin et al. 2007; Bauswein et al. 2013; Sekiguchi et al. 2016). This dominant blue component is also seen in early-time optical spectra (Nicholl et al. 2017). By contrast, the low velocity red $\mathrm{KN}$ component in our three-component model could originate from the dynamically ejected tidal tails in the equatorial plane of the binary (e.g., Rosswog et al. 1999; Hotokezaka et al. 2013 ), in which case the relatively high ejecta mass $\approx 0.01 M_{\odot}$ suggests an asymmetric mass ratio of the merging binary $(q \lesssim 0.8$; Hotokezaka et al. 2013)

In both multi-component models we find that the $\kappa \approx 3 \mathrm{~cm}^{2} \mathrm{~g}^{-1}$ ejecta dominates by mass. The lower velocity of this component suggests an origin in the post-merger accretion disk outflow. Our inferred ejecta mass is consistent with that expected for a massive $\sim 0.1 M_{\odot}$ torus (e.g., Just et al. 2015; Siegel \& Metzger 2017). Similarly, the disk outflow composition is predicted to be dominated by $Y_{e} \sim 0.3$ matter that produces the $\kappa \approx 3 \mathrm{~cm}^{2} \mathrm{~g}^{-1}$ component of the $\mathrm{KN}$ emission (Tanaka et al. 2017) as we observe. The fitted opacity indicates that the hyper-massive neutron star remnant is relatively short-lived ( $\sim 30 \mathrm{~ms}$; Fernández \& Metzger 2013; Just et al. 2015; Kasen et al. 2015). We additionally find that in both models the total kinetic energy is roughly $(1-2) \times 10^{51} \mathrm{erg}$.

The fact that our multi-component models fit the data well provides strong evidence for the production of both light and heavy $r$-process nuclei, addressing one of the long-standing mysteries in astrophysics (Burbidge et al. 1957; Cameron 
1957). We quantify this statement by comparing our blue and red ejecta masses to those necessary to reproduce the Milky Way (MW) $r$-process production rate. For heavy $r$-process elements (red $\mathrm{KN}$ ), the MW inferred production rate is $\dot{M}_{\mathrm{rp}, \mathrm{A} \gtrsim 140} \approx 10^{-7} M_{\odot} \mathrm{yr}^{-1}$ (Bauswein et al. 2014). For light $r$-process elements (blue $\mathrm{KN}$ ), the production rate is $\dot{M}_{\mathrm{rp}, \mathrm{A}} \gtrsim 100 \approx 7 \times 10^{-7} M_{\odot} \mathrm{yr}^{-1}$ (Qian 2000). Using a conservative estimate on the local BNS merger rate estimated by Abbott et al. (2016), $R_{0} \approx 1000 \mathrm{Gpc}^{-3} \mathrm{yr}^{-1}$, and a volume density of MW-like galaxies of $\approx 0.01 \mathrm{Mpc}^{-3}$, we estimate the MW rate of $\mathrm{KN}$ as $R_{\mathrm{MW}} \approx 100 \mathrm{Myr}^{-1}$. Using this MW rate, we find that the average ejecta mass for a red $\mathrm{KN}$ is $\dot{M}_{\mathrm{rp}, \mathrm{A} \gtrsim 140} / R_{\mathrm{MW}} \approx 0.001 M_{\odot}$ and for a blue $\mathrm{KN}$ it is $\dot{M}_{\mathrm{rp}, \mathrm{A} \gtrsim 100} / R_{\mathrm{MW}} \approx 0.007 M_{\odot}$. These order-of-magnitude estimates are smaller than our inferred ejecta masses for this event, although the discrepancy can potentially be mitigated when properly taking into account the fraction of $r$-process materials that remains in a gas phase in the ISM and galactic halo. Nevertheless, this exercise suggests that BNS mergers can reproduce the $r$-process yields found in the MW and may be a dominant source of cosmic $r$-process nucleosynthesis.

\section{Discussions and Conclusions}

We have presented a comprehensive UV, optical, and NIR data set for the first electromagnetic counterpart to be associated with a gravitational wave event. Analysis of these data reveals that the emission is due to an $r$-process-powered KN consisting of both "blue" and "purple/red" ejecta components. Models with ${ }^{56} \mathrm{Ni}$ heating, Fe-peak opacities, or a single component of $r$-process opacity fail to match the observations.

Our models indicate that the total ejecta mass is $\approx 0.05 M_{\odot}$, with a high velocity $(v \approx 0.3 c)$ blue component and a slower $(v \approx 0.1-0.2 c)$ purple/red component. The presence of both components and the relatively large ejecta mass suggests that binary neutron star mergers (like GW170817) dominate the cosmic $r$-process nucleosynthesis.

The data presented in this paper (and others in this series) represent by far the best observations of an $r$-process powered $\mathrm{KN}$, and it is remarkable how well the observations match theoretical models. This event also marks the true beginning of joint GW-EM multi-messenger astronomy. We expect that this event will serve as a benchmark for future efforts to model and understand the behavior of these transients, and for the first time allow the development of data-driven KN models. The next Advanced LIGO/Virgo observing run (starting in Fall 2018) is expected to detect many more BNS events (Abbott et al. 2016c). The follow-up of these events will provide further understanding of the ubiquity of the features seen in this event, the relationship between event and host properties, and place even stronger constraints on $r$-process enrichment from BNS mergers.

The Berger Time-Domain Group at Harvard is supported in part by the NSF through grants AST-1411763 and AST1714498, and by NASA through grants NNX15AE50G and NNX16AC22G. P.S.C. is grateful for support provided by the NSF through the Graduate Research Fellowship Program, grant
DGE 1144152. V.A.V. acknowledges support by the National Science Foundation through a Graduate Research Fellowship. D.A.B. is supposed by NSF award PHY-1707954. We thank the University of Copenhagen, DARK Cosmology Centre, and the Niels Bohr International Academy for hosting R.J.F. during the discovery of GW170817/SSS17a, where he was participating in the Kavli Summer Program in Astrophysics, "Astrophysics with gravitational wave detections." This program was supported by the Kavli Foundation, Danish National Research Foundation, the Niels Bohr International Academy, and the DARK Cosmology Centre. The UCSC group is supported in part by NSF grant AST-1518052, the Gordon \& Betty Moore Foundation, the Heising-Simons Foundation, generous donations from many individuals through a UCSC Giving Day grant, and from fellowships from the Alfred P. Sloan Foundation and the David and Lucile Packard Foundation to R.J.F. E.B. acknowledges financial support from the European Research Council (ERC-StG-335936, CLUSTERS). D.K. and E.Q. were funded in part by the Gordon and Betty Moore Foundation through Grant GBMF5076.

We are grateful for the heroic efforts of the entire staff at Gemini-South in order to continue obtaining observations of this target in evening twilight at high airmass as the object was setting.

This project used data obtained with the Dark Energy Camera (DECam), which was constructed by the Dark Energy Survey (DES) collaboration. Funding for the DES Projects has been provided by the DOE and NSF (USA), MISE (Spain), STFC (UK), HEFCE (UK), NCSA (UIUC), KICP (U. Chicago), CCAPP (Ohio State), MIFPA (Texas A\&M), CNPQ, FAPERJ, FINEP (Brazil), MINECO (Spain), DFG (Germany), and the collaborating institutions in the Dark Energy Survey, which are Argonne Lab, UC Santa Cruz, University of Cambridge, CIEMAT-Madrid, University of Chicago, University College London, DES-Brazil Consortium, University of Edinburgh, ETH Zürich, Fermilab, University of Illinois, ICE (IEEC-CSIC), IFAE Barcelona, Lawrence Berkeley Lab, LMU München, and the associated Excellence Cluster Universe, University of Michigan, NOAO, University of Nottingham, Ohio State University, University of Pennsylvania, University of Portsmouth, SLAC National Lab, Stanford University, University of Sussex, and Texas A\&M University.

Based in part on observations at Cerro Tololo InterAmerican Observatory, National Optical Astronomy Observatory (NOAO Prop. ID: 2017B-0110, PI: E. Berger), which is operated by the Association of Universities for Research in Astronomy (AURA) under a cooperative agreement with the National Science Foundation.

Based in part on observations obtained at the Gemini Observatory (Program IDs GS-2017B-Q-8 and GS-2017B-DD4; PI: Chornock), which is operated by the Association of Universities for Research in Astronomy, Inc., under a cooperative agreement with the NSF on behalf of the Gemini partnership: the National Science Foundation (United States), the National Research Council (Canada), CONICYT (Chile), Ministerio de Ciencia, Tecnología e Innovación Productiva (Argentina), and Ministério da Ciência, Tecnologia e Inovação (Brazil). 
Appendix Summary of Photometry

Table 2

Summary of Photometry

\begin{tabular}{|c|c|c|c|c|}
\hline Telescope & Instrument & Filter & MJD & Mag [AB] \\
\hline Blanco & DECam & $i$ & 0.4745 & $17.48 \pm 0.03$ \\
\hline Blanco & DECam & $z$ & 0.4752 & $17.59 \pm 0.03$ \\
\hline Swift & UVOT & $M 2$ & 0.627 & $21.14 \pm 0.23$ \\
\hline Swift & UVOT & $W 1$ & 0.634 & $19.53 \pm 0.12$ \\
\hline Swift & UVOT0 & $U$ & 0.639 & $18.20 \pm 0.09$ \\
\hline Swift & UVOT & $W 2$ & 0.643 & $20.76 \pm 0.20$ \\
\hline Swift & UVOT0 & $U$ & 0.981 & $18.90 \pm 0.17$ \\
\hline Swift & UVOT0 & $U$ & 1.043 & $18.98 \pm 0.16$ \\
\hline Blanco & DECam & $Y$ & 1.4478 & $17.32 \pm 0.03$ \\
\hline Blanco & DECam & $z$ & 1.4485 & $17.59 \pm 0.02$ \\
\hline Blanco & DECam & $i$ & 1.4492 & $17.78 \pm 0.02$ \\
\hline Blanco & DECam & $r$ & 1.4499 & $18.04 \pm 0.02$ \\
\hline Blanco & DECam & $g$ & 1.4506 & $18.66 \pm 0.03$ \\
\hline Blanco & DECam & $u$ & 1.4512 & $19.94 \pm 0.05$ \\
\hline Gemini-South & FLAMINGOS-2 & $H$ & 1.471 & $17.63 \pm 0.10$ \\
\hline Gemini-South & FLAMINGOS-2 & $H$ & 2.439 & $17.71 \pm 0.09$ \\
\hline Blanco & DECam & $Y$ & 2.4461 & $17.77 \pm 0.03$ \\
\hline Blanco & DECam & $z$ & 2.4472 & $18.18 \pm 0.03$ \\
\hline Blanco & DECam & $i$ & 2.4479 & $18.38 \pm 0.03$ \\
\hline Blanco & DECam & $r$ & 2.4486 & $19.03 \pm 0.03$ \\
\hline Blanco & DECam & $g$ & 2.4492 & $20.21 \pm 0.05$ \\
\hline Blanco & DECam & $u$ & 2.4515 & $22.26 \pm 0.16$ \\
\hline Blanco & DECam & $Y$ & 3.4541 & $18.05 \pm 0.03$ \\
\hline Blanco & DECam & $z$ & 3.4551 & $18.56 \pm 0.03$ \\
\hline Blanco & DECam & $u$ & 3.4556 & $23.06 \pm 0.32$ \\
\hline Blanco & DECam & $i$ & 3.4558 & $18.73 \pm 0.03$ \\
\hline Blanco & DECam & $r$ & 3.4564 & $19.29 \pm 0.04$ \\
\hline Blanco & DECam & $g$ & 3.4571 & $20.93 \pm 0.08$ \\
\hline Gemini-South & FLAMINGOS-2 & $H$ & 4.445 & $17.92 \pm 0.10$ \\
\hline Blanco & DECam & $Y$ & 4.4467 & $18.35 \pm 0.03$ \\
\hline Blanco & DECam & $z$ & 4.4491 & $18.81 \pm 0.03$ \\
\hline Blanco & DECam & $i$ & 4.4516 & $19.22 \pm 0.03$ \\
\hline Blanco & DECam & $r$ & 4.4552 & $20.25 \pm 0.05$ \\
\hline Blanco & DECam & $g$ & 4.4624 & $21.73 \pm 0.11$ \\
\hline Blanco & DECam & $Y$ & 5.4460 & $18.83 \pm 0.18$ \\
\hline Blanco & DECam & $z$ & 5.4484 & $19.17 \pm 0.11$ \\
\hline Blanco & DECam & $i$ & 5.4508 & $19.55 \pm 0.18$ \\
\hline Blanco & DECam & $r$ & 5.4545 & $20.79 \pm 0.24$ \\
\hline Blanco & DECam & $g$ & 5.462 & $>20.80$ \\
\hline Blanco & DECam & $Y$ & 6.4458 & $19.06 \pm 0.31$ \\
\hline Blanco & DECam & $r$ & 6.457 & $>19.60$ \\
\hline Blanco & DECam & $g$ & 6.468 & $>20.67$ \\
\hline Gemini-South & FLAMINGOS-2 & $H$ & 7.438 & $18.79 \pm 0.14$ \\
\hline Blanco & DECam & $Y$ & 7.4448 & $19.44 \pm 0.05$ \\
\hline Blanco & DECam & $z$ & 7.4509 & $19.89 \pm 0.05$ \\
\hline Blanco & DECam & $i$ & 7.4533 & $20.54 \pm 0.05$ \\
\hline Blanco & DECam & $r$ & 7.4581 & $21.23 \pm 0.11$ \\
\hline Blanco & DECam & $g$ & 7.469 & $>22.19$ \\
\hline Blanco & DECam & $Y$ & 8.4446 & $20.06 \pm 0.07$ \\
\hline Gemini-South & FLAMINGOS-2 & $H$ & 8.452 & $19.22 \pm 0.18$ \\
\hline Blanco & DECam & $z$ & 8.4543 & $20.40 \pm 0.06$ \\
\hline Blanco & DECam & $i$ & 8.4591 & $20.72 \pm 0.06$ \\
\hline Blanco & DECam & $r$ & 8.4688 & $21.95 \pm 0.18$ \\
\hline Blanco & DECam & $Y$ & 9.4457 & $20.78 \pm 0.11$ \\
\hline Gemini-South & FLAMINGOS-2 & $H$ & 9.449 & $19.62 \pm 0.15$ \\
\hline Blanco & DECam & $z$ & 9.4659 & $21.19 \pm 0.07$ \\
\hline Blanco & DECam & $i$ & 9.4712 & $21.37 \pm 0.06$ \\
\hline$H S T$ & WFC3/IR & F110W & 9.753 & $20.57 \pm 0.04$ \\
\hline$H S T$ & WFC3/IR & F160W & 9.768 & $19.89 \pm 0.04$ \\
\hline$H S T$ & WFC3/UVIS & F336W & 9.819 & $26.92 \pm 0.27$ \\
\hline
\end{tabular}

Table 2

(Continued)

\begin{tabular}{llccc}
\hline \hline Telescope & Instrument & Filter & MJD & Mag [AB] \\
\hline$H S T$ & ACS/WFC & F475W & 9.905 & $23.95 \pm 0.06$ \\
$H S T$ & ACS/WFC & F625W & 9.969 & $22.88 \pm 0.07$ \\
$H S T$ & ACS/WFC & F775W & 10.032 & $22.35 \pm 0.08$ \\
$H S T$ & ACS/WFC & F850LP & 10.045 & $21.53 \pm 0.05$ \\
Blanco & DECam & $Y$ & 10.4462 & $21.67 \pm 0.21$ \\
Gemini-South & FLAMINGOS-2 & $K_{s}$ & 10.449 & $18.43 \pm 0.25$ \\
Gemini-South & FLAMINGOS-2 & $H$ & 10.453 & $20.04 \pm 0.15$ \\
Blanco & DECam & $z$ & 10.4583 & $22.06 \pm 0.13$ \\
Blanco & DECam & $i$ & 10.4715 & $22.38 \pm 0.10$ \\
Gemini-South & FLAMINGOS-2 & $K_{s}$ & 11.455 & $19.03 \pm 0.17$ \\
Gemini-South & FLAMINGOS-2 & $K_{s}$ & 12.447 & $19.42 \pm 0.16$ \\
Gemini-South & FLAMINGOS-2 & $K_{s}$ & 13.441 & $19.63 \pm 0.23$ \\
Gemini-South & FLAMINGOS-2 & $K_{s}$ & 14.446 & $19.90 \pm 0.21$ \\
Gemini-South & FLAMINGOS-2 & $K_{s}$ & 15.447 & $20.13 \pm 0.25$ \\
Gemini-South & FLAMINGOS-2 & $K_{s}$ & 16.446 & $20.43 \pm 0.30$ \\
Gemini-South & FLAMINGOS-2 & $K_{s}$ & 18.450 & $20.84 \pm 0.26$ \\
\hline
\end{tabular}

Note. Summary of photometry from Section 2. Dates are give in days relative to the time of the GW trigger (MJD $=57982.529)$. Photometry is not corrected for extinction. Limits are given at the $3 \sigma$ level. Error bars are given at the $1 \sigma$ level.

\section{ORCID iDs}

P. S. Cowperthwaite (1) https://orcid.org/0000-00022478-6939

E. Berger @ https://orcid.org/0000-0002-9392-9681

V. A. Villar (i) https://orcid.org/0000-0002-5814-4061

M. Nicholl (i) https://orcid.org/0000-0002-2555-3192

W. Fong @i https://orcid.org/0000-0002-7374-935X

R. Margutti (i) https://orcid.org/0000-0003-4768-7586

M. Soares-Santos (i) https://orcid.org/0000-0001-6082-8529

\section{References}

Abbott, B. P., Abbott, R., Abbott, T., et al. 2016, ApJL, 832, L21

Abbott, B. P., Abbott, R., Abbott, T. D., et al. 2016a, PhRvL, 116, 061102 Abbott, B. P., Abbott, R., Abbott, T. D., et al. 2016b, PhRvL, 116, 241103

Abbott, B. P., Abbott, R., Abbott, T. D., et al. 2016c, LRR, 19, 1

Abbott, B. P., Abbott, R., Abbott, T. D., et al. 2017a, PhRvL, 118, 221101

Abbott, B. P., Abbott, R., Abbott, T. D., et al. 2017b, ApJL, https://doi.org/ $10.3847 / 2041-8213 /$ aa91c9

Alexander, K., Berger, E., Fong, W., et al. 2017, ApJL, https://doi.org/ 10.3847/2041-8213/aa905d

Allam, S., Annis, J., Berger, E., et al. 2017, GCN, 21530

Barnes, J., \& Kasen, D. 2013, ApJ, 775, 18

Bauswein, A., Goriely, S., \& Janka, H.-T. 2013, ApJ, 773, 78

Bauswein, A., Pulpillo, R. A., Janka, H.-T., \& Goriely, S. 2014, ApJL, 795, L9

Becker, A. 2015, HOTPANTS: High Order Transform of PSF ANd Template Subtraction, Astrophysics Source Code Library, ascl:1504.004

Bianco, F. B., Modjaz, M., Hicken, M., et al. 2014, ApJS, 213, 19

Blackburn, L., Briggs, M. S., Broida, J., et al. 2017, GCN, 21506

Blanchard, P. K., Berger, E., Fong, W., et al. 2017, ApJL, https://doi.org/ 10.3847/2041-8213/aa9055

Brown, P. J., Holland, S. T., Immler, S., et al. 2009, AJ, 137, 4517

Burbidge, E. M., Burbidge, G. R., Fowler, W. A., \& Hoyle, F. 1957, RvMP, 29, 547

Cameron, A. G. W. 1957, PASP, 69, 201

Cenko, S. B., Emery, S. W. K., Campana, S., et al. 2017, GCN, 21572

Chambers, K. C., Magnier, E. A., Metcalfe, N., et al. 2016, arXiv:1612.05560

Chornock, R., Berger, E., Kasen, D., et al. 2017, ApJL, https://doi.org/ 10.3847/2041-8213/aa905c

Coulter, D. A., Kilpatrick, C. D., Siebert, M. A., et al. 2017a, Sci, https://doi. org/10.1126/science.aap9811

Coulter, D. A., Kilpatrick, C. D., Siebert, M. R., et al. 2017b, GCN, 21529 
Eikenberry, S., Bandyopadhyay, R., Bennett, J. G., et al. 2012, Proc. SPIE, 8446, 84460I

Evans, P. A., Cenko, S., Kennea, D. A., et al. 2017a, Sci, https://doi.org/ 10.1126/science.aap9580

Evans, P. A., Kennea, J. A., Breeveld, A. A., et al. 2017b, GCN, 21550

Fernández, R., \& Metzger, B. D. 2013, MNRAS, 435, 502

Flaugher, B., Diehl, H. T., Honscheid, K., et al. 2015, AJ, 150, 150

Folatelli, G., Phillips, M. M., Burns, C. R., et al. 2010, AJ, 139, 120

Freedman, W. L., Madore, B. F., Gibson, B. K., et al. 2001, ApJ, 553, 47

Galbany, L., Hamuy, M., Phillips, M. M., et al. 2016, AJ, 151, 33

Gehrels, N., Chincarini, G., Giommi, P., et al. 2004, ApJ, 611, 1005

Gelman, A., Hwang, J., \& Vehtari, A. 2014, Stat. Comput., 24, 997

Gelman, A., \& Rubin, D. B. 1992, StaSc, 457

Goldstein, A., Veres, P., Burns, E., et al. 2017a, ApJL, https://doi.org/ $10.3847 / 2041-8213 /$ aa8f41

Goldstein, A., Veres, P., von Kienlin, A., et al. 2017b, GCN, 21528

Guillochon, J., Nicholl, M., Villar, V. A., et al. 2017, ApJS, submitted

Hotokezaka, K., Kiuchi, K., Kyutoku, K., et al. 2013, PhRvD, 87, 024001

Just, O., Bauswein, A., Pulpillo, R. A., Goriely, S., \& Janka, H.-T. 2015, MNRAS, 448, 541

Kasen, D., Badnell, N., \& Barnes, J. 2013, ApJ, 774, 25

Kasen, D., Fernández, R., \& Metzger, B. D. 2015, MNRAS, 450, 1777

Li, L.-X., \& Paczyński, B. 1998, ApJL, 507, L59

LIGO Scientific Collaboration, Virgo Collaboration, Abbott, R., et al. 2017, arXiv: 1709.09660

LIGO Scientific Collaboration and Virgo Collaboration 2017a, PhRvL, https://doi.org/10.1103/PhysRevLett.119.161101

LIGO Scientific Collaboration and Virgo Collaboration 2017b, GCN, 21509

LIGO Scientific Collaboration and Virgo Collaboration 2017c, GCN, 21513

Margutti, R., Berger, E., Fong, W., et al. 2017, ApJL, https://doi.org/ 10.3847/2041-8213/aa9057

Metzger, B., Martínez-Pinedo, G., Darbha, S., et al. 2010, MNRAS, 406, 2650

Metzger, B. D. 2017, LRR, 20, 3

Metzger, B. D., \& Berger, E. 2012, ApJ, 746, 48

Metzger, B. D., \& Fernández, R. 2014, MNRAS, 441, 3444
Modjaz, M., Liu, Y. Q., Bianco, F. B., \& Graur, O. 2016, ApJ, 832, 108

Nicholl, M., Guillochon, J., \& Berger, E. 2017, arXiv:1706.00825

Nicholl, M., Berger, E., Kasen, D., et al. 2017, ApJL, https://doi.org/ 10.3847/2041-8213/aa9029

Nugent, P., Kim, A., \& Perlmutter, S. 2002, PASP, 114, 803

Oechslin, R., Janka, H.-T., \& Marek, A. 2007, å, 467, 395

Qian, Y.-Z. 2000, ApJL, 534, L67

Rest, A., Scolnic, D., Foley, R. J., et al. 2014, ApJ, 795, 44

Rest, A., Stubbs, C., Becker, A. C., et al. 2005, ApJ, 634, 1103

Roberts, L. F., Kasen, D., Lee, W. H., \& Ramirez-Ruiz, E. 2011, ApJL, 736, L21

Roming, P. W. A., Kennedy, T. E., Mason, K. O., et al. 2005, SSRv, 120, 95

Rosswog, S., Liebendörfer, M., Thielemann, F.-K., et al. 1999, A\&A, 341, 499

Savchenko, V., Ferrigno, C., Kuulkers, E., et al. 2017a, ApJL, https://doi. org/10.3847/2041-8213/aa8f94

Savchenko, V., Mereghetti, S., Ferrigno, C., et al. 2017b, GCN, 21507

Schechter, P. L., Mateo, M., \& Saha, A. 1993, PASP, 105, 1342

Schlafly, E. F., \& Finkbeiner, D. P. 2011, ApJ, 737, 103

Scolnic, D., Casertano, S., Riess, A., et al. 2015, ApJ, 815, 117

Sekiguchi, Y., Kiuchi, K., Kyutoku, K., Shibata, M., \& Taniguchi, K. 2016, PhRvD, 93, 124046

Siegel, D. M., \& Metzger, B. D. 2017, arXiv:1705.05473

Soares-Santos, M., Holz, D. E., Annis, J., et al. 2017, ApJL, https://doi.org/10.3847/2041-8213/aa9059

Stetson, P. B. 1987, PASP, 99, 191

Tanaka, M., \& Hotokezaka, K. 2013, ApJ, 775, 113

Tanaka, M., Kato, D., Gaigalas, G., et al. 2017, arXiv:1708.09101

Valenti, S., Sand, D. J., Yang, S., et al. 2017, ApJL, https://doi.org/10.3847/ 2041-8213/aa8edf

Villar, V. A., Berger, E., Metzger, B. D., \& Guillochon, J. 2017, arXiv:1707. 08132

von Kienlin, A., Meegan, C., \& Goldstein, A. 2017, GCN, 21520

Watanabe, S. 2010, J. Mach. Learn. Res., 11, 3571

Wollaeger, R. T., Korobkin, O., Fontes, C. J., et al. 2017, arXiv:1705.07084

Yang, S., Valenti, S., Sand, D., et al. 2017, GCN, 21531 